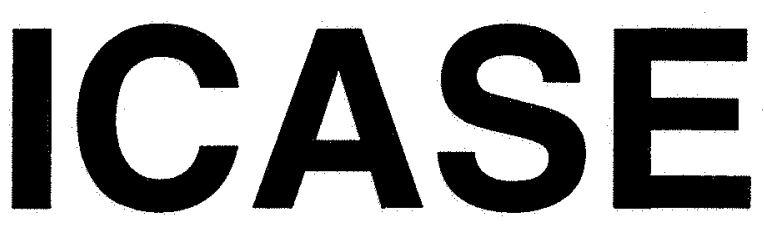

\title{
ANALYTICAL THEORY OF THE DESTRUCTION TERMS IN DISSIPATION RATE TRANSPORT EQUATIONS
}

\section{Robert Rubinstein} Ye Zhou

NASA Contract No. NAS1-19480

March 1996

Institute for Computer Applications in Science and Engineering NASA Langley Research Center Hampton, VA 23681-0001

Operated by Universities Space Research Association

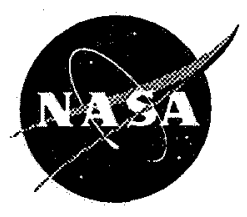

National Aeronautics and Space Administration

Langley Research Center Hampton, Virginia 23681-0001 


\title{
ANALYTICAL THEORY OF THE DESTRUCTION TERMS IN DISSIPATION RATE TRANSPORT EQUATIONS
}

\author{
Robert Rubinstein and Ye Zhou* \\ Institute for Computer Applications in Science and Engineering \\ MS 132C NASA Langley Research Center \\ Hampton, VA 23681
}

\begin{abstract}
Modeled dissipation rate transport equations are often derived by invoking various hypotheses to close correlations in the corresponding exact equations. D. C. Leslie suggested that these models might be derived instead from Kraichnan's wavenumber space integrals for inertial range transport power. This suggestion is applied to the destruction terms in the dissipation rate equations for incompressible turbulence, buoyant turbulence, rotating incompressible turbulence, and rotating buoyant turbulence. Model constants like $C_{\varepsilon 2}$ are expressed as integrals; convergence of these integrals implies the absence of Reynolds number dependence in the corresponding destruction term. The dependence of $C_{\varepsilon 2}$ on rotation rate emerges naturally; sensitization of the modeled dissipation rate equation to rotation is not required. A buoyancy related effect which is absent in the exact transport equation for temperature variance dissipation, but which sometimes improves computational predictions, also arises naturally. Both the presence of this effect and the appropriate time scale in the modeled transport equation depend on whether Bolgiano or Kolmogorov inertial range scaling applies. A simple application of these methods leads to a preliminary dissipation rate equation for rotating buoyant turbulence.
\end{abstract}

* This research was supported by the National Aeronautics and Space Administration under NASA Contract No. NAS1-19480 while the authors were in residence at the Institute for Computer Applications in Science and Engineering (ICASE), NASA Langley Research Center, Hampton, VA. 


\section{Introduction}

Despite widespread agreement in the turbulence modeling community about the basic structure of the dissipation rate transport equation, questions remain about its theoretical grounding. The central problem remains to justify the absence of $O\left(\operatorname{Re}_{T}^{1 / 2}\right)$ terms in this equation, the possible existence of which is suggested by elementary arguments. ${ }^{1}$ More basic difficulties arise when there are coupled fluctuating fields as in buoyant turbulence, or imposed time scales as in rotating turbulence. In these cases, even the form of the appropriate transport equation is doubtful. Thus, it has been suggested ${ }^{2}$ that the transport equation for dissipation of temperature variance $\varepsilon_{\theta}$ should contain a term related to the buoyant production of turbulence energy, although this requirement does not follow from the exact transport equation for $\varepsilon_{\theta}$. Similarly, although rotation is well known to have effects which must be reflected in the dissipation rate transport equation, such effects do not occur explicitly in the exact equation for $\varepsilon$. These effects are therefore sometimes introduced by sensitizing ${ }^{3}$ the transport equation to rotation in various ways.

Attempts to derive the $\varepsilon$ transport equation from analytical theories have been only partially successful. In a preliminary discussion of two equation modeling based on the direct interaction approximation ${ }^{4}$ (DIA), Leslie ${ }^{5}$ observed that whereas the transport equation for turbulence kinetic energy followed in principle by integrating the DIA correlation equation over all wavevectors, the analogous treatment of the response equation, which should lead to a transport equation for a time scale, does not suggest itself as readily. Leslie asserted that dimensional analysis alone fixes the functional form of the $\varepsilon$ equation, but this statement neglects the possibility of $R_{T}$ dependence. A subsequent analytical investigation of the dissipation rate transport equation by Yoshizawa ${ }^{6}$ led to $O\left(\operatorname{Re}_{T}^{1 / 2}\right)$ terms and to dependence on the energy spectrum in the dissipation range. A later analysis by Yakhot and Orszag ${ }^{7}$ was criticized by Smith and Reynolds ${ }^{8}$ and then reformulated by Smith and Yakhot. ${ }^{9}$ This work identified the absence of $O\left(R e_{T}^{1 / 2}\right)$ terms with a cancellation of divergences in the renormalization group formulation of the dissipation rate equation. This point of view must be contrasted with the usual one in the modeling literature, namely that $O\left(R e_{T}^{1 / 2}\right)$ terms are absent because certain production and dissipation terms in the exact transport equation for dissipation rate cancel. The failure of this cancellation, unbalanced vortex stretching, has been analyzed by Speziale and Bernard, ${ }^{10}$ and of course 
leads to explicit $R e_{T}$ dependent terms.

These investigations of the $\varepsilon$ transport equation follow the usual approach in turbulence modeling of closing correlations in the exact equation for $\dot{\varepsilon}=\langle\nu \nabla \dot{\mathbf{u}} \cdot \nabla \mathbf{u}\rangle$. A different approach is taken by Hamba, ${ }^{11}$ namely that a transport equation for eddy viscosity is obtained by Yoshizawa's two-scale direct interaction approximation ${ }^{12,13}$ and the transport equation for $\varepsilon$ is then inferred from this equation and a $K$ equation. The present analysis is based instead on Leslie's suggestion ${ }^{5}$ that the $\varepsilon$ transport equation be derived from Kraichnan's integrals ${ }^{14}$ for inertial range transport power. Leslie's original discussion was general enough to include effects of production and turbulent diffusion; the present analysis is restricted to issues accessible to a homogeneous theory. Applied to incompressible turbulence, this procedure leads to an integral for $C_{\varepsilon 2}$; the convergence of this integral at high wavenumbers is equivalent to the absence of $R_{T}$ dependence of the destruction term. A modeled dissipation rate equation for rotating turbulence is derived by substituting rotation-dependent field descriptors in Kraichnan's integrals. To illustrate the general method, the phenomenological theory of rotating turbulence of $\mathrm{Zhou}^{15}$ is applied. The dependence of $C_{\varepsilon 2}$ on rotation emerges naturally, and even provides a result very similar to models proposed by Speziale. ${ }^{16}$ Buoyant turbulence is investigated by writing the analogous integral for $\varepsilon_{\theta}$. The analysis leads naturally to the buoyancy dependent term suggested in Ref. 2. Straighforward application of the arguments of Ref. 15 to buoyant turbulence leads to a preliminary dissipation rate equation for rotating buoyant turbulence. The present approach to the dissipation rate equation, like that of Hamba and Yoshizawa, offers an alternative to the standard derivations. Application of this approach to production and diffusion terms will be reported later.

\section{The dissipation rate equation in incompressible turbulence}

It is generally accepted that inertial range spectral transport power must be balanced by viscous dissipation; accordingly, equating dissipation to the inertial range transfer integral of Kraichnan, ${ }^{14}$

$$
\varepsilon=\frac{i}{2}\left[I^{-}\left(k^{\prime}\right)-I^{+}\left(k^{\prime}\right)\right] P_{i m n}(\mathbf{k})<u_{m}(\mathbf{p}) u_{n}(\mathbf{q}) u_{i}(-\mathbf{k})>
$$

where

$$
\begin{aligned}
P_{i m n}(\mathbf{k}) & =k_{m} P_{i n}(\mathbf{k})+k_{n} P_{i m}(\mathbf{k}) \\
P_{i j}(\mathbf{k}) & =\delta_{i j}-k_{i} k_{j} / k^{2}
\end{aligned}
$$


and the integration operators are defined by

$$
\begin{aligned}
I^{+}\left(k^{\prime}\right) & =\int_{k^{\prime}}^{2 k^{\prime}} d \mathbf{k} \int_{p, q \leq k^{\prime} ; \mathbf{k}=\mathbf{p}+\mathbf{q}} d \mathbf{p} d \mathbf{q} \\
I^{-}\left(k^{\prime}\right) & =\int_{0}^{k^{\prime}} d \mathbf{k} \int_{p, q \geq k^{\prime} ; \mathbf{k}=\mathbf{p}+\mathbf{q}} d \mathbf{p} d \mathbf{q}
\end{aligned}
$$

Time arguments in the Fourier amplitudes $u_{i}$ are understood. Differentiate Eq. (1) with respect to time, substitute the equations of motion for the $\dot{u}$ terms, and apply the quasinormal hypothesis to obtain

$$
\begin{aligned}
\dot{\varepsilon} & =\frac{i}{2}\left[I^{-}\left(k^{\prime}\right)-I^{+}\left(k^{\prime}\right)\right] P_{i m n}(\mathbf{k})\left\{<\dot{u}_{m}(\mathbf{p}) u_{n}(\mathbf{q}) u_{i}(-\mathbf{k})>+<u_{m}(\mathbf{p}) \dot{u}_{n}(\mathbf{q}) u_{i}(-\mathbf{k})>\right. \\
& \left.+<u_{m}(\mathbf{p}) u_{n}(\mathbf{q}) \dot{u}_{i}(-\mathbf{k})>\right\} \\
& =\frac{1}{4}\left[I^{-}\left(k^{\prime}\right)-I^{+}\left(k^{\prime}\right)\right]\left\{2 P_{i m n}(\mathbf{k}) P_{m r s}(\mathbf{p}) Q_{n s}(\mathbf{q}) Q_{i r}(\mathbf{k})\right. \\
& \left.-P_{i m n}(\mathbf{k}) P_{i r s}(\mathbf{k}) Q_{n s}(\mathbf{q}) Q_{m r}(\mathbf{p})\right\}
\end{aligned}
$$

In turbulence with an isotropic inertial range, the correlation tensor takes the form

$$
Q_{i j}(\mathbf{k})=Q(k) P_{i j}(\mathbf{k})
$$

where the correlation function $Q$ is related to the energy spectrum by

$$
4 \pi k^{2} Q(k)=E(k)
$$

The integrals in Eq. (2) will be evaluated by substituting the Kolmogorov spectrum

$$
E(k)=C_{K} \varepsilon^{2 / 3} k^{-5 / 3}
$$

The absence of $R e_{T}$ dependence will follow from the convergence of these integrals at high wavenumbers: if the integrals diverged at either high or low wavenumbers, it would be necessary to substitute instead a truncated Kolmogorov spectrum such as

$$
E(k)= \begin{cases}C_{K} \varepsilon^{2 / 3} k^{-5 / 3} & \text { if } k_{0} \leq k \leq k_{d} \\ 0 & \text { if } k \leq k_{0} \text { or } k \geq k_{d}\end{cases}
$$

and investigate the dependence on the cutoffs $k_{0}$ and $k_{d}$. Dependence on $k_{d}$, due to divergence at high wavenumbers, would imply Reynolds number dependence through the 
relation $R e_{T}=\left(k_{d} / k_{0}\right)^{4 / 3}$. The convergence of the integrals Eq. (2) would prove the locality of $\dot{\varepsilon}$, namely that $\dot{\varepsilon}$ is a property of the inertial range alone.

Substitute the isotropic forms Eqs. (3)-(5) in Eq. (2). The convergence of each term will be investigated separately.

There are two $I^{-}$terms:

$$
\begin{aligned}
& \mathcal{I}_{1}^{-}=I^{-} P_{i m n}(\mathbf{k}) P_{m r s}(\mathbf{p}) P_{n s}(\mathbf{q}) P_{i r}(\mathbf{k}) Q(q) Q(k) \\
& \mathcal{I}_{2}^{-}=I^{-} P_{i m n}(\mathbf{k}) P_{i r s}(\mathbf{k}) P_{n s}(\mathbf{q}) P_{m r}(\mathbf{p}) Q(p) Q(q)
\end{aligned}
$$

If $p \rightarrow \infty$ with $\mathbf{k}$ fixed, the triangle condition $\mathbf{k}=\mathbf{p}+\mathbf{q}$ implies that $q \rightarrow p$. First consider the integral $\mathcal{I}_{1}^{-}$defined in Eq. (7). Substitute the leading order result $\mathbf{q}=\mathbf{p} ; \mathcal{I}_{1}^{-}$vanishes to this order because it is of odd order in $\mathbf{p}$. Expand the $\mathbf{q}$ dependent terms to next order in $k / p$. Then $\mathcal{I}_{1}^{-}$is of the order

$$
\begin{gathered}
\mathcal{I}_{1}^{-} \sim \int_{0}^{k^{\prime}} E(k) d k \int_{k^{\prime}}^{\infty} d p E(p) k p(k / p)=\int_{0}^{k^{\prime}} d k k^{2} E(k) \int_{k^{\prime}}^{\infty} d p E(p) \\
\sim\left(k^{\prime}\right)^{4 / 3}\left(k^{\prime}\right)^{-2 / 3}=\left(k^{\prime}\right)^{2 / 3}
\end{gathered}
$$

For the term $\mathcal{I}_{2}^{-}$of Eq. (8), the scaling is found directly to be

$$
\mathcal{I}_{2}^{-} \sim \int_{0}^{k^{\prime}} k^{4} d k \int_{k^{\prime}}^{\infty} d p p^{2} p^{-22 / 3} \sim\left(k^{\prime}\right)^{5}\left(k^{\prime}\right)^{-13 / 3} \sim\left(k^{\prime}\right)^{2 / 3}
$$

To investigate the convergence of the $I^{+}$terms, note first that since the wavevectors are all bounded from above in these integrals, there is no question of high wavenumber divergence, so consider their convergence at low wavenumbers. It will be convenient to rewrite the $I^{+}$terms symmetrically as

$$
\begin{aligned}
\mathcal{I}^{+}=I^{+} P_{i m n}(\mathbf{k})\{ & P_{m r s}(\mathbf{p}) P_{n s}(\mathbf{q}) P_{i r}(\mathbf{k}) Q(q) Q(k)+P_{m r s}(\mathbf{q}) P_{n s}(\mathbf{p}) P_{i r}(\mathbf{k}) Q(p) Q(k) \\
& \left.-P_{i r s}(\mathbf{k}) P_{n s}(\mathbf{q}) P_{m r}(\mathbf{p}) Q(q) Q(p)\right\}
\end{aligned}
$$

It suffices to check convergence in the limit $q \rightarrow 0$. The triangle condition implies that also $\mathbf{p} \rightarrow \mathbf{k}$. Substitute the lowest order result $\mathbf{p}=\mathbf{k}$ in first and third terms in Eq. (11) to obtain the integrand

$$
P_{i m n}(\mathbf{k}) P_{n s}(\mathbf{q})\left\{P_{m r s}(\mathbf{k}) P_{i r}(\mathbf{k})-P_{i r s}(\mathbf{k}) P_{m r}(\mathbf{k})\right\} \equiv 0
$$


Thus, this potential divergence of the $\mathbf{p}$ integration cancels. The term of first order in $\mathbf{q}$ vanishes after spherical integration. The second order term will lead to the scaling

$$
\int_{k^{\prime}}^{2 k^{\prime}} d k E(k) \int_{0}^{k^{\prime}} d q E(q) k\left(q^{2} / k\right) \sim \int_{k^{\prime}}^{2 k^{\prime}} E(k) d k \int_{0}^{k^{\prime}} d q q^{2} E(q) \sim\left(k^{\prime}\right)^{2 / 3}
$$

Note that the second term in Eq. (11) also scales this way: the leading order term vanishes because it is of odd order and the term of next order scales as above. We conclude that

$$
\mathcal{I}^{+} \sim\left(k^{\prime}\right)^{2 / 3}
$$

Adding Eqs. (9), (10), and (12),

$$
\dot{\varepsilon} \sim-\mathcal{I}^{+}+\mathcal{I}_{1}^{-}+\mathcal{I}_{2}^{-} \sim \varepsilon^{4 / 3}\left(k^{\prime}\right)^{2 / 3}
$$

So far, the scale $k^{\prime}$ is arbitrary and $\dot{\varepsilon}$ has been evaluated assuming an infinite Kolmogorov inertial range. To apply these results to single point turbulence modeling, it is appropriate to replace the infinite Kolmogorov range by a spectrum with a finite Kolmogorov range exending over scales $k_{0}<k<k_{d}$. The convergence of the integrals under consideration implies that they are weak functions of the actual energy spectrum in the low and high wavenumber regions $k<k_{0}$ and $k>k_{d}$. Accordingly, we will make the analytically simplest choice, that the spectrum vanishes outside this range, as in Eq. (6).

To fix the scale $k^{\prime}$, we reason that in single point turbulence modeling, $\varepsilon$ is the power input into the inertial range, so that $\dot{\varepsilon}$ should be evaluated at $k^{\prime}=k_{0}$, corresponding to the largest inertial range scale. If a model of this type were to be applied in a large eddy simulation, $k^{\prime}$ would be equated instead to the inverse filter size. In the context of single point modeling, the choice $k^{\prime}=k_{0}$ is also consistent with the choice of $K / \varepsilon$ as a time scale, since $\varepsilon / K \sim \varepsilon^{1 / 3} k_{0}^{2 / 3}$ is the Komogorov inertial range frequency evaluated at $k_{0}$. The turbulence kinetic energy $K$ is given in the infinite Reynolds number limit $k_{0} \rightarrow 0$ by

$$
K=\int_{k_{0}}^{\infty} d k E(k)=\frac{3}{2} C_{K} \varepsilon^{2 / 3} k_{0}^{-2 / 3}
$$

Substituting Eqs. (3), (4), and (6) in Eq. (2) and setting $k^{\prime}=k_{0}$, the cutoffs cause all terms to vanish except for the last term in $I^{-}$, so that

$$
\dot{\varepsilon}=-\frac{1}{4} \int_{0}^{k_{0}} d \mathbf{k} P_{i m n}(\mathbf{k}) P_{i r s}(\mathbf{k}) \int_{\mathbf{k}=\mathbf{p}+\mathbf{q} ; p, q \geq k_{0}} d \mathbf{p} d \mathbf{q} P_{m r}(\mathbf{p}) P_{n s}(\mathbf{q}) Q(p) Q(q)
$$


Eq. (13) with $k^{\prime}=k_{0}$ and Eqs. (14)-(15) then imply that

$$
\dot{\varepsilon}=-C_{\varepsilon 2} \frac{\varepsilon^{2}}{K}
$$

Although the constant $C_{\varepsilon 2}$ could be evaluated from Eq. (15), our concern here is only to derive the forms of the relevant models. Note that the demonstration that the integrals converge is necessary to prove the proportionality of $\dot{\varepsilon}$ to $\varepsilon^{2} / K$ because dimensional analysis alone cannot rule out dependence on $R e_{T}$.

\section{The dissipation rate equation in rotating turbulence}

Imposed time scale effects like rotation can be included in this analysis provided that time scale dependent field descriptors are known. Thus, a rotation dependent equation for $\dot{\varepsilon}$ will follow by substituting rotation dependent expressions for the correlation $Q_{i j}$ in Eq. (2). Appropriate expressions appear in the recent work of Mahalov and Zhou. ${ }^{17}$ This procedure could help address theoretical questions concerning anisotropy of spectral transfer in rotating turbulence. In order to evaluate the overall effect of rotation on the dissipation rate equation, we will apply Zhou's simplified isotropic theory of rotating turbulence ${ }^{15}$ based on a phenomenological prescription for the rotation dependent decorrelation time. Naturally, geometric effects like two-dimensionalization are not accessible to such a theory; a more complete theory would begin with anisotropic expressions and then evaluate shell averages.

The theory of Ref. 15 predicts that for large rotation rates $\Omega$, the energy spectrum is given by

$$
E(p, \Omega)=C_{K}^{\Omega}(\varepsilon|\Omega|)^{1 / 2} p^{-2}
$$

where the modified Kolmogorov constant could be computed from the flux integral for the $k^{-2}$ energy spectrum and $k^{0}$ time scale postulated ${ }^{15}$ for the strong rotation limit. For general rotation rates,

$$
E(p, \Omega)=Z(p, \Omega) E(p)
$$

where $E(p)$ is the rotation independent Kolmogorov spectrum and the rotation dependent correction $Z$ is given in Ref. 15. Substituting Eqs. (18), (3), and (4) in Eq. (2) leads to an expression for $\dot{\varepsilon}$ which cannot be evaluated analytically; the resulting integral could only 
be given in tabular form as a function of $\Omega$. Since the rotation dependent spectrum of $E q$. (18) is only approximate, it is reasonable to simplify the theory further.

We will follow a procedure which has now become widespread, ${ }^{18}$ namely to evaluate $\dot{\varepsilon}$ in the strong rotation limit, and then bridge the strong and weak rotation limits by an $a d$ hoc interpolation. Substituting the strong rotation spectrum of Eq. (17) in Eq. (2),

$$
\begin{gathered}
\dot{\varepsilon}=-\frac{1}{4}\left(C_{K}^{\Omega}\right)^{2} \varepsilon|\Omega| \int_{0}^{k_{0}} d \mathbf{k} P_{i m n}(\mathbf{k}) P_{i r s}(\mathbf{k}) \\
\int_{\mathbf{k}=\mathbf{p}+\mathbf{q} ; p, q \geq k_{0}} d \mathbf{p} d \mathbf{q} P_{m r}(\mathbf{p}) P_{n s}(\mathbf{q})(p q)^{-2}
\end{gathered}
$$

The integral in Eq. (19) can be shown to be convergent and independent of $k_{0}$; therefore

$$
\dot{\varepsilon}=-C_{\varepsilon 2}^{\Omega}|\Omega| \varepsilon
$$

in the strong rotation limit.

A consequence of this model is the complete suppression of decay of turbulence in the strong rotation limit: the solution of Eq. (20) with the energy equation $\dot{K}=-\varepsilon$ and initial conditions $K=K_{0}, \varepsilon=\varepsilon_{0}$ when $t=0$ is

$$
\begin{aligned}
K / K_{0} & =1+\frac{\varepsilon_{0}}{C \Omega K_{0}}\left[e^{-C \Omega t}-1\right] \\
\varepsilon / \varepsilon_{0} & =e^{-C \Omega t}
\end{aligned}
$$

where $C=C_{\varepsilon 2}^{\Omega}$. The long time limit $\varepsilon=0$ indicates the complete suppression of energy transfer in this model.

A simple formula which interpolates between the strong rotation limit defined by Eq. (20) and the case of no rotation defined by Eq. (16) is the Bardina ${ }^{19}$ correction

$$
\dot{\varepsilon}=-C_{\varepsilon 2} \frac{\varepsilon^{2}}{K}\left\{1+\frac{C_{\varepsilon 2}^{\Omega}}{C_{\varepsilon 2}} \frac{|\Omega| K}{\varepsilon}\right\}
$$

But unless helicity is explicitly included in the theory, the corrections for small $\Omega$ must be independent of the sign of $\Omega$ and hence quadratic to lowest order. A simple expression with this additional property is

$$
\dot{\varepsilon}=-C_{\varepsilon 2} \frac{\varepsilon^{2}}{K}\left\{1+\left[\frac{C_{\varepsilon 2}^{\Omega}}{C_{\varepsilon 2}} \frac{\Omega K}{\varepsilon}\right]^{2}\right\}^{1 / 2}
$$


Speziale ${ }^{16}$ has recently obtained a model with the same form as Eq. (21) by an entirely different argument beginning from stress transport models. Simulations ${ }^{20}$ of rotating decaying turbulence using Eq. (21) suggest that fully satisfactory comparison with DNS data may require low Reynolds number corrections of the model. ${ }^{16}$

\section{The dissipation rate equation in buoyant turbulence}

In buoyant turbulence, the additional spectral flux $\varepsilon_{\theta}$ appears; the analog of Eq. (1) is

$$
\varepsilon_{\theta}=i\left\{I^{-}-I^{+}\right\} k_{m}<T(\mathbf{p}) u_{m}(\mathbf{q}) T(-\mathbf{k})>
$$

where $T$ denotes the temperature. The correlations in buoyant turbulence are defined by

$$
\begin{aligned}
Q^{T}(\mathbf{k}) & =<T(\mathbf{k}) T(-\mathbf{k})> \\
Q_{r}^{h}(\mathbf{k}) & =<T(\mathbf{k}) u_{r}(-\mathbf{k})> \\
Q_{i j}^{u}(\mathbf{k}) & =<u_{i}(\mathbf{k}) u_{j}(-\mathbf{k})>
\end{aligned}
$$

Differentiating Eq. (22) with respect to time, following the procedure of Sect. II,

$$
\begin{aligned}
\dot{\varepsilon}_{\theta} & =i\left\{I^{-}-I^{+}\right\} k_{m}\left\{<\dot{T}(\mathbf{p}) u_{m}(\mathbf{q}) T(-\mathbf{k})>+<T(\mathbf{p}) \dot{u}_{m}(\mathbf{q}) T(-\mathbf{k})>\right. \\
+ & \left.<T(\mathbf{p}) u_{m}(\mathbf{q}) \dot{T}(-\mathbf{k})>\right\} \\
& =\left\{I^{-}-I^{+}\right\}\left[\mathcal{T}^{\mathbf{1}}(\mathbf{k}, \mathbf{p}, \mathbf{q})-g \mathcal{T}^{2}(\mathbf{k}, \mathbf{p}, \mathbf{q})\right]
\end{aligned}
$$

where the direct interaction approximation gives

$$
\begin{aligned}
\mathcal{T}^{1} & =\frac{1}{4}\left\{k_{m} p_{n} Q^{T}(\mathbf{k}) Q_{m n}^{u}(\mathbf{q})+k_{m} p_{n} Q_{m}^{h}(\mathbf{q}) Q_{n}^{h}(\mathbf{k})\right. \\
& +k_{m} P_{m r s}(\mathbf{q}) Q_{r}^{h}(\mathbf{k}) Q_{s}^{h}(\mathbf{p}) \\
& \left.-k_{m} k_{n} Q_{m}^{h}(\mathbf{p}) Q_{n}^{h}(\mathbf{q})-k_{m} k_{n} Q^{T}(\mathbf{p}) Q_{m n}^{u}(\mathbf{q})\right\}
\end{aligned}
$$

and

$$
\begin{aligned}
\mathcal{T}^{2} & =\frac{1}{4} k_{m} P_{m 3}(\mathbf{q})\left\{p_{r} \Theta(\mathbf{p}, \mathbf{k}, \mathbf{q})\left[Q_{r}^{h}(\mathbf{k}) Q^{T}(\mathbf{q})+Q^{T}(\mathbf{k}) Q_{r}^{h}(\mathbf{q})\right]\right. \\
& +q_{r} \Theta(\mathbf{q}, \mathbf{k}, \mathbf{p})\left[Q_{r}^{h}(\mathbf{k}) Q^{T}(\mathbf{p})+Q^{T}(\mathbf{k}) Q_{r}^{h}(\mathbf{p})\right] \\
& \left.-k_{r} \Theta(\mathbf{k}, \mathbf{p}, \mathbf{q})\left[Q^{T}(\mathbf{p}) Q_{r}^{h}(\mathbf{q})+Q^{T}(\mathbf{q}) Q_{r}^{h}(\mathbf{p})\right]\right\}
\end{aligned}
$$


and gravity acts in the 3 direction. The constant $g$ denotes the product of the acceleration of gravity and the thermal expansion coefficient of the fluid. The $\mathcal{T}^{2}$ terms originate from the $\dot{u}$ term in Eq. (24); the quantity $\Theta$ in Eq. (26) is a wavenumber-dependent time scale.

Recent theoretical arguments ${ }^{21,22}$ suggest that buoyant turbulence can exhibit Bolgiano scaling ${ }^{23}$ in which the spectra corresponding to $Q^{T}, Q^{h}, Q^{u}$ are

$$
\begin{aligned}
& E^{u}=C_{K}^{\theta} g^{4 / 5} \varepsilon_{\theta}^{2 / 5} k^{-11 / 5} \\
& E^{h}=C_{h}^{\theta} g^{1 / 5} \varepsilon_{\theta}^{3 / 5} k^{-9 / 5} \\
& E^{T}=C_{T}^{\theta} g^{-2 / 5} \varepsilon_{\theta}^{4 / 5} k^{-7 / 5}
\end{aligned}
$$

In this scaling, the time scale $\Theta$ has the form

$$
\Theta(\mathbf{k}, \mathbf{p}, \mathbf{q})=[\eta(k)+a \eta(p)+\eta(q)]^{-1}
$$

where

$$
\eta(k)=C_{D}^{\theta} g^{2 / 5} \varepsilon_{\theta}^{1 / 5} k^{2 / 5}
$$

and $a$ is the inverse turbulent Prandtl number for free convection. A preliminary attempt to compute the inertial range constants $a, C_{K}^{\theta}, C_{h}^{\theta}, C_{T}^{\theta}$, and $C_{D}^{\theta}$ appears in Ref. 24. Bolgiano scaling should describe buoyant flows, or at least scales of motion, in which the gravitational coupling dominates nonlinearity, although the occurence of this scaling in Rayleigh-Benard convection remains controversial. ${ }^{25}$ If instead, the gravitational coupling is small compared to the nonlinearity, then the temperature is a passive scalar with a source of fluctuations; Kolmogorov scaling applies, in which

$$
\begin{aligned}
& E^{u}=C_{K} \varepsilon^{2 / 3} k^{-5 / 3} \\
& E^{h}=0 \\
& E^{T}=C_{B} \varepsilon_{\theta} \varepsilon^{-1 / 3} k^{-5 / 3}
\end{aligned}
$$

Kolmogorov scaling applies to locally isotropic turbulence; accordingly, the heat transfer spectrum $E^{h}$ vanishes in Eq. (30). Bolgiano scaling applies to anisotropic turbulence in which the direction of gravity is distinguished; this permits a nonvanishing $E^{h}$ in Eq. (27).

The dissipation rate equation for each type of scaling will be treated separately. 


\section{A. Bolgiano scaling}

In evaluating the integral Eq. (24) with Eqs. (25)-(27), the isotropic contribution to the energy transfer will be evaluated by setting

$$
\begin{aligned}
Q^{T}(\mathbf{k}) & =Q^{T}(k) \\
Q_{r}^{h}(\mathbf{k}) & =Q^{h}(k) P_{r 3}(\mathbf{k}) \\
Q_{i j}^{u}(\mathbf{k}) & =Q^{u}(k) P_{i j}(\mathbf{k})
\end{aligned}
$$

When these substitutions are made, the index 3 always appears in pairs. Thus, to evaluate the isotropic part of the energy transfer, it is convenient to sum over this index. This procedure leads, as in Sects. II and III, to an integral for $\dot{\varepsilon}_{\theta}$; the proof that this integral converges is similar to the proof in Sect. II and therefore need not be given. The terms $\mathcal{T}^{1}$ scale after integration as

$$
\left\{I^{-}-I^{+}\right\} \mathcal{T}^{1} \sim g^{2 / 5} \varepsilon_{\theta}^{6 / 5}\left(k^{\prime}\right)^{2 / 5}
$$

Integrating $E^{T}$ from Eq. (27) over a Bolgiano spectrum with lower cutoff $k_{0}$ leads to

$$
K_{\theta}=\frac{5}{2} C_{T}^{\theta} g^{-2 / 5} \varepsilon_{\theta}^{4 / 5} k_{0}^{-2 / 5}
$$

thus, in terms of single point quantities, setting $k^{\prime}=k_{0}$

$$
\begin{gathered}
\left\{I^{-}-I^{+}\right\} \mathcal{I}^{1}=-\frac{1}{4} \int_{0}^{k_{0}} d \mathbf{k} k_{m} k_{n} \int_{\mathbf{k}=\mathbf{p}+\mathbf{q} ; p, q \geq k_{0}} d \mathbf{p} d \mathbf{q} \times \\
\left\{\frac{1}{3} Q_{m p}^{h}(\mathbf{p}) Q_{n p}^{h}(\mathbf{q})+Q^{T}(\mathbf{p}) Q_{m n}^{u}(\mathbf{q})\right\}
\end{gathered}
$$

or in view of Eqs. (31)-(32),

$$
\left\{I^{+}-I^{-}\right\} \mathcal{T}^{1}=-C_{\varepsilon 2}^{\theta} \frac{\varepsilon_{\theta}}{K_{\theta}} \varepsilon_{\theta}
$$

Analogous treatment of the $\mathcal{T}^{2}$ terms gives

$$
\left\{I^{-}-I^{+}\right\} \mathcal{T}^{2} \sim g^{-3 / 5} \varepsilon_{\theta}^{6 / 5} k_{0}^{2 / 5}
$$

Integrating the Bolgiano spectra $E^{h}$ and $E^{u}$ of Eq. (27),

$$
\begin{aligned}
& H=\frac{5}{4} C_{h}^{\theta} g^{1 / 5} \varepsilon_{\theta}^{3 / 5} k_{0}^{-4 / 5} \\
& K=\frac{5}{6} C_{K}^{\theta} g^{4 / 5} \varepsilon_{\theta}^{2 / 5} k_{0}^{-6 / 5}
\end{aligned}
$$


where the heat transfer $H$ is the single point moment $\left.H=-<u_{3} T\right\rangle$. Substituting Eq. (36) in Eq. (35),

$$
\left\{I^{-}-I^{+}\right\} \mathcal{T}^{2}=C_{\varepsilon 5}^{\theta} \frac{\varepsilon_{\theta}}{K} H
$$

Combining the results of Eqs. (34) and (37),

$$
\dot{\varepsilon}_{\theta}=-C_{\varepsilon 2}^{\theta} \frac{\varepsilon_{\theta}}{K_{\theta}} \varepsilon_{\theta}-C_{\varepsilon 5}^{\theta} g \frac{\varepsilon_{\theta}}{K}<u_{3} T>
$$

which agrees with the model proposed in Ref. 2 from which the nomenclature for the model constants is taken.

\section{B. Kolmogorov scaling}

Substituting the Kolmogorov spectra Eq. (30) in Eqs. (24)-(26) leads to

$$
\left\{I^{-}-I^{+}\right\} \mathcal{T}^{1} \sim-\varepsilon_{\theta} \varepsilon^{-1 / 3} \varepsilon^{2 / 3} k_{0}^{2 / 3}
$$

The term $\mathcal{T}^{2}$ vanishes since $Q^{h}=0$; therefore,

$$
\dot{\varepsilon}_{\theta}=-C_{\varepsilon 4}^{\theta} \frac{\varepsilon}{K} \varepsilon_{\theta}
$$

Comparison of Eqs. (38) and (39) reveals that Bolgiano and Kolmogorov scaling have fundamentally different implications for modeling buoyant turbulence. First, the $g$ dependent term of Eq. (38) is absent in Eq. (39). More fundamentally, the time scale in Eq. (38) is the thermal time scale $K_{\theta} / \varepsilon_{\theta}$ whereas the time scale in Eq. (39) is the velocity time scale $K / \varepsilon$. The results for different scaling laws are combined in the model of Ref. 2 , in which the destruction terms are

$$
\dot{\varepsilon}_{\theta}=-C_{\varepsilon 2}^{\theta} \frac{\varepsilon_{\theta}}{K_{\theta}} \varepsilon_{\theta}-C_{\varepsilon 4}^{\theta} \frac{\varepsilon}{K} \varepsilon_{\theta}-C_{\varepsilon 5}^{\theta} g \frac{\varepsilon_{\theta}}{K}<u_{3} T>
$$

A model of this form is not inconsistent with the present theory, since Bolgiano and Kolmogorov scaling can both occur in the same problem at different inertial range scales. ${ }^{21,22}$ However, particular problems can be dominated by only one type of scaling: computational experience indicates ${ }^{26}$ that the $g$ dependent term in Eq. (40) improves computational predictions for some problems but degrades them in others; this difference may reflect dominance in these problems by different scaling laws. Future work must clarify how the existence of these two scaling laws should be reflected in turbulence models. 


\section{Rotating buoyant turbulence}

A modeled transport equation for $\varepsilon_{\theta}$ in rotating buoyant turbulence follows from Eqs. (24)-(26) by substituting rotation-dependent field quantities $Q^{T}, Q_{i}^{h}$ and $Q_{i j}^{u}$; the calculation does not differ in principle from that of Sect. III for rotating incompressible turbulence. As in Sects. III and IV, the present analysis will be limited to a simplified isotropic analysis which treats only the overall effect of this type of coupling; the subtle geometric effects which can arise if the rotation axis does not coincide with the direction of gravity are inaccessible to this type of theory. The discussion will be limited to Bolgiano scaling.

The approximate analysis of Bolgiano scaling in Ref. 24 expresses $E^{h}$ and $E^{u}$ in terms of $E^{T}$ by

$$
E^{h} \sim g \Theta E^{T} \quad E^{u} \sim(g \Theta)^{2} E^{T}
$$

where $\Theta$ is a time scale. A rotation dependent spectrum $E^{T}$ can be found from the DIA flux balance for Bolgiano scaling. For the purpose of computing scalings, this balance takes the form

$$
\varepsilon_{\theta} \sim g^{2} k^{4} \Theta E^{T} E^{u}
$$

Following Zhou ${ }^{15}$, assume that $\Theta \sim|\Omega|^{-1}$ in the limit of strong rotation. Substituting $E^{u}$ from Eq. (41) in Eq. (42),

$$
E^{T}=C_{T}^{\Omega \theta} g^{-1}|\Omega|^{3 / 2} \varepsilon_{\theta}^{1 / 2} k^{-2}
$$

Then Eq. (41) implies

$$
\begin{aligned}
& E^{h}=C_{h}^{\Omega \theta}|\Omega|^{1 / 2} \varepsilon_{\theta}^{1 / 2} k^{-2} \\
& E^{u}=C_{K}^{\Omega \theta} g|\Omega|^{-1 / 2} \varepsilon_{\theta}^{1 / 2} k^{-2}
\end{aligned}
$$

It is crucial that the DIA flux integral, given in simplified form as Eq. (42), converges when the spectra of Eqs. (43)-(44) are substituted in it. The convergence of the flux integral is easily checked; only because of this convergence can the scaling law of Eq. (43) be inferred from Eq. (42).

Substitution of Eqs. (42) and (44) in Eq. (24) leads as in Sect. III to the strong rotation limit

$$
\dot{\varepsilon}_{\theta}=-C_{\varepsilon 2}^{\Omega \theta}|\Omega| \varepsilon_{\theta}
$$


An $a d$ hoc bridge between the weak and strong rotation limits can be constructed as in Sect. III. A possible form is

$$
\begin{aligned}
\dot{\varepsilon}_{\theta} & =-C_{\varepsilon 2}^{\theta} \frac{\varepsilon_{\theta}}{K_{\theta}} \varepsilon_{\theta}\left\{1+\left[\frac{C_{\varepsilon 2}^{\theta \Omega}}{C_{\varepsilon 2}^{\theta}} \frac{\Omega K_{\theta}}{\varepsilon_{\theta}}\right]^{2}\right\}^{1 / 2} \\
& -C_{\varepsilon 5}^{\theta} g \frac{\varepsilon_{\theta}}{K}<u_{3} T>\left\{1+\left[\frac{C_{\varepsilon 2}^{\theta \Omega}}{C_{\varepsilon 5}^{\theta}} \frac{\Omega K}{g \varepsilon_{\theta} H}\right]^{2}\right\}^{1 / 2}
\end{aligned}
$$

\section{Conclusions}

We summarize the models obtained for the destruction terms in dissipation rate transport equations:

A. Incompressible turbulence

$$
\dot{\varepsilon}=-C_{\varepsilon 2} \frac{\varepsilon^{2}}{K}
$$

where $C_{\varepsilon 2}$ can be computed from Eq. (15). Convergence of this integral at high wavenumbers implies that $C_{\varepsilon 2}$ does not depend on turbulent Reynolds number $R_{T}$.

B. Rotating turbulence

For rapid rotation,

$$
\dot{\varepsilon}=-C_{\varepsilon 2}^{\Omega}|\Omega| \varepsilon
$$

where $C_{\varepsilon 2}^{\Omega}$ is found from Eq. (19). A possible interpolation formula between the weak and strong rotation limits is

$$
\dot{\varepsilon}=-C_{\varepsilon 2} \frac{\varepsilon^{2}}{K}\left\{1+\left[\frac{C_{\varepsilon 2}^{\Omega}}{C_{\varepsilon 2}} \frac{\Omega K}{\varepsilon}\right]^{2}\right\}^{1 / 2}
$$

\section{Buoyant turbulence}

For Bolgiano scaling,

$$
\dot{\varepsilon}_{\theta}=-C_{\varepsilon 2}^{\theta} \frac{\varepsilon_{\theta}}{K_{\theta}} \varepsilon_{\theta}-C_{\varepsilon 5}^{\theta} g \frac{\varepsilon_{\theta}}{K}<u_{3} T>
$$

For Kolmogorov scaling

$$
\dot{\varepsilon}_{\theta}=-C_{\varepsilon 4}^{\theta} \frac{\varepsilon}{K} \varepsilon_{\theta}
$$

The time scale for Bolgiano scaling is $\varepsilon_{\theta} / K_{\theta}$; the time scale for Kolmogorov scaling is $\varepsilon / K$. The buoyancy effect present for Bolgiano scaling is absent for Kolmogorov scaling. 
Bolgiano and Kolmogorov scaling are believed to occur together in general flows; this suggests that the general dissipation rate model could combine the results for both scalings as in Eq. (40). ${ }^{2}$ However, a complete model must provide for flows dominated by either scaling law alone.

\section{Rotating buoyant turbulence}

In the strong rotation limit, for Bolgiano scaling

$$
\dot{\varepsilon}_{\theta}=-C_{\varepsilon 2}^{\theta \Omega}|\Omega| \varepsilon_{\theta}
$$

As in the case of rotating incompressible turbulence, an ad hoc expression interpolating the weak and strong rotation limits can be constucted.

\section{References}

1. H. Tennekes and J. L. Lumley, A first course in turbulence, MIT Press (1972).

2. K. Hanjalic, "Achievements and limitations in modelling and computation of buoyant turbulent flows and heat transfer," 10th International Heat Transfer Conference (1993).

3. K. Hanjalic and B. Launder, "Sensitizing the dissipation equation to irrotational strains," ASME J. Fluids Eng. 102, 34 (1980).

C. G. Speziale, R. Raj, and T. B. Gatski, "Modeling the dissipation rate in rotating turbulent flows," in Studies in Turbulence, T. B. Gatski, S. Sarkar, and C. G. Speziale eds., Springer (1992).

4. R. H. Kraichnan, "The structure of isotropic turbulence at very high Reynolds number," J. Fluid Mech. 5, 497 (1959).

5. D. C. Leslie, Modern developments in the theory of turbulence, Oxford University Press (1972).

6. A. Yoshizawa, "A statistical investigation of the transport equation for energy dissipation in shear turbulence," J. Phys. Soc. Japan 51, 1983 (1982).

7. V. Yakhot and S. A. Orszag, "Renormalization group theory of turbulence," J. Sci. Comput. 13 (1986).

8. L. M. Smith and W. C. Reynolds, "On the Yakhot-Orszag renormalization group method for deriving turbulence statistics and models," Phys. Fluids A 2, 364 (1992). 
9. V. Yakhot and L. M. Smith, "The renormalization group, the $\epsilon$-expansion, and derivation of turbulence models," J. Sci. Comput. 7, 35 (1992).

10. C. G. Speziale and P. S. Bernard, "The energy decay in self-preserving isotropic turbulence revisited," J. Fluid Mech. 241, 645 (1992).

11. F. Hamba, "Statistical investigation of the energy dissipation equation in shear turbulence," J. Phys. Soc. Japan 56, 3771 (1987).

12. A. Yoshizawa, "A statistically derived system of equations for turbulent shear flows," Phys. Fluids 28, 59 (1985).

13. A. Yoshizawa, "Statistical analysis of the deviation of the Reynolds stress from its eddy viscosity representation," Phys. Fluids 27, 1377 (1984).

14. R. H. Kraichnan, "Inertial range transfer in two and three dimensional turbulence," J. Fluid Mech. 47 (1971).

15. Y. Zhou, "A phenomenological treatment of rotating turbulence," Phys. Fluids 7 $2092(1995)$.

16. C. G. Speziale, private communication.

17. A. Mahalov and Y. Zhou, "Analytical and phenomenological studies of rotating turbulence," ICASE report 95-72 (1995).

18. V. Yakhot, S. A. Orszag, S. Thangam, T. B. Gatski, and C. G. Speziale, "Development of turbulence models for shear flows by a double expansion technique," Phys. Fluids A 7, (1993).

19. J. Bardina, J. H. Ferziger, and R. S. Rogallo, "Effect of rotation on isotropic turbulence: computation and modeling," J. Fluid Mech. 154, 321 (1985).

20. T. B. Gatski, private communication.

21. V. S. L'vov and G. E. Falkovich, "Conservation laws and two flux spectra of hydrodynamic convective turbulence," Physica D 57 (1992).

22. S. Grossmann and V. S. L'vov, "Crossover of spectral scaling in thermal turbulence," Phys. Rev. E 47, 4161 (1993).

23. R. Bolgiano, "Turbulent spectra in a stably stratified atmosphere," J. Geophys. Res. 64, 2226 (1959).

24. R. Rubinstein, "Inertial range dynamics in Boussinesq turbulence," ICASE report 96-2, (1996). 
25. E. Siggia, "High Rayleigh number convection," Annu. Rev. Fluid Mech 26, 137 (1994).

26. B. Younis, private communication 


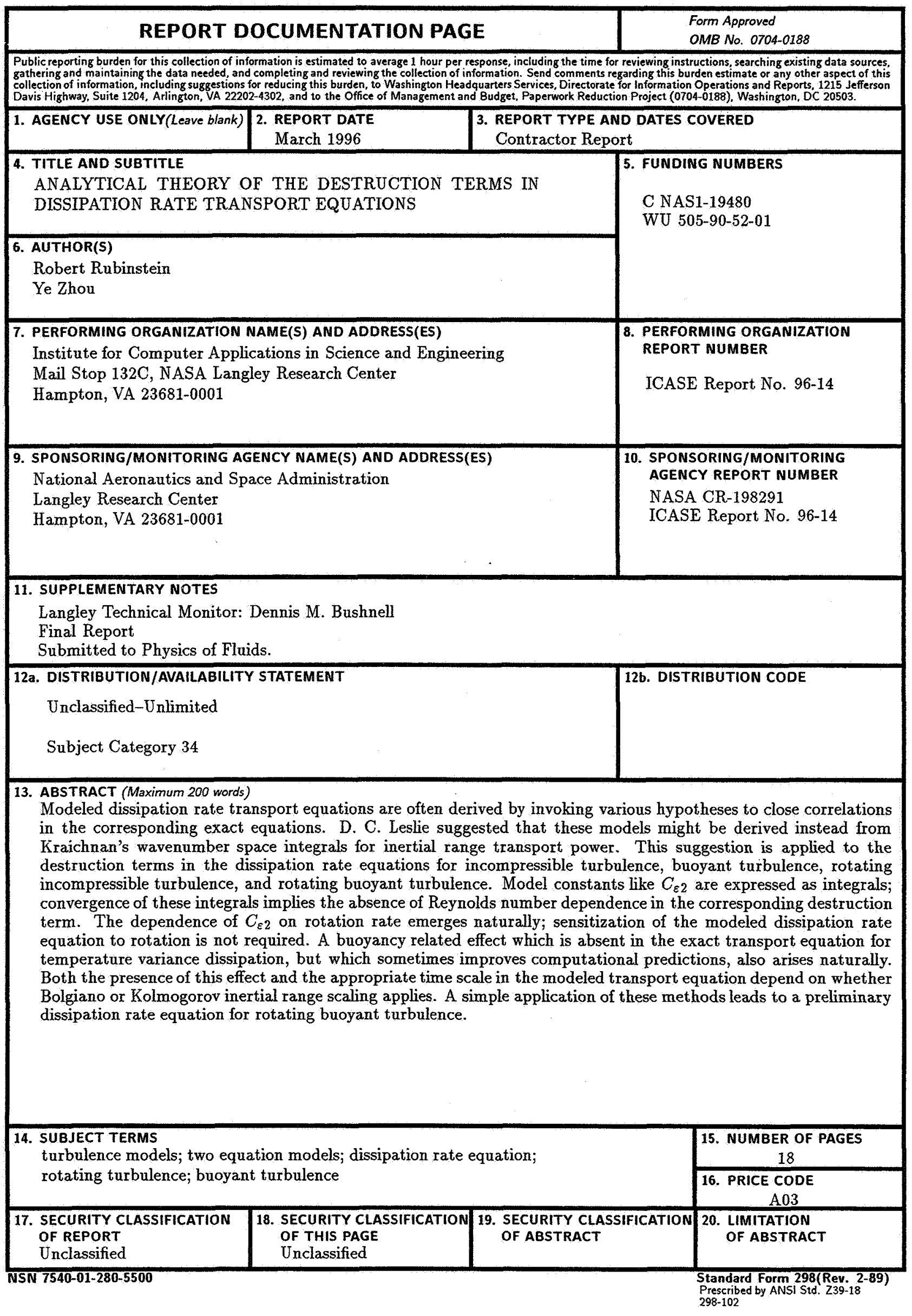

